\title{
On the Kellogg method and its variants for finding of eigenvalues and eigenfunctions of linear self-adjoint operators
}

\author{
J. KoLOMx́
}

(Prof. Dr. F. A. Willers zu seinem 100. Geburtstag gewidmet)

Es werden die Kelloggsche Methode, die Birgersche Methode und die modifizierte Birgersche Prozedur zur Bestinmung von Eigenwerten und Eigenvektoren für lineare nichtnegative selbstadjungierte Operatoren in Hilbertschen Räumen untersucht.

В статье рассматриваютсп методы Келлогга и Биргера и модифицированый метоп Биргера для определения собственных значениі и собственшых функцй линейых самосопряжённых операторов в пространствах Гинберта.

The Kellogg method, Birger's method and its modified Birger procedure for determination of eigenvalues and eigenvectors of linear nonnegative and self-adjoint operators are investigated.

The Kellogg method, Birger's method and the modified Birger procedure for determination of eigenvalues and eigenvectors of linear nonnegative and self-adjoint opeartors are investigated.

\section{Introduction}

In this note we show that the methods mentioned above converge even in the case, when the starting approximation is only different from zero. We also give the estimate for the distance of eigenvalues of two different operators. Moreover, some conditions for the starting approximation equivalent to that of Theoren 1 are established.

Let us recall that BIRGER [2] has proposed his method without any mathematica] justification. However, he has found (on engineering problems) that his method has some advantages in comparison with the other ones, see also MARCHUK [15], where a similar observation has been done on the ground of physical ideas. The corivergence proofs of the Birger and the modified Birger methods for compact symmetrizable operators were given in $[7,8]$ under the condition that the starting approximation is not orthogonal to annihilator of the certain eigenspace. Later BüCKN ER [3] independently proposed the same method as Birger and proved its convergence for the class of linear and nonlinear compact operators having some decomposition properties. Further results in these topics have been obtained under various hypotheses by MAREK [14], PETRYShyN [16] and the author [9-12]. We refer the reader also for instance to $[1,4,13,16]$ for some further and related methods. Let us note that the Birger method and the BückNer [3] results have been applied for instance by Briger [2], Marchuk [15], Conivay and Thomas [5] and 'Thomas [18]. 


\section{Convergence theorems}

Let $X$ he a real Hilhert space with the scalar product $\langle\cdot, \cdot\rangle, A: X \rightarrow X$ a linear nonnegative and self-adjoint operator defined on $X$. By saying that $A$ is non-negative we mean that $\langle A u, u\rangle \geqq 0$ for each $u \in X$. Since $A$ is self-adjoint and is defined on all of $X, A$ is bounded by the closed graph theorem. The spectrum $\sigma(A)$ of $A$ lies in the segment $\left[m, \lambda_{1}\right]$, where $m=\inf \{\langle A u, u\rangle:\|u\|=1\}, m \neq 0$, and $\lambda_{1}=\sup \{\langle A u, u\rangle:\|u\|$ $=1\}$. The symbol $\left\{E_{\lambda}\right\}$ stands for the spectral resolution of identity corresponding to the self-adjoint operator $A$.

Under the assumptions stated above the following approximate methods for determination of the eigenvalues and eigenvectors will be considered:

(i) the Kellogg method:

$$
\alpha_{n+1}=\left\|A u_{n}\right\|_{1}^{1}, \quad u_{n+1}=\alpha_{n+1}^{-1} A u_{n} \quad(n=0,1,2, \ldots),
$$

where the starting approximation $u_{0} \in X$ is such that $u_{0} \oplus$ ker $A,\left\|u_{0}\right\|=1$. According to our assumptions $\alpha_{n}>0$ and $\left\|u_{n}\right\|=1$ for each $n$;

(ii) the modified Birger method:

$$
\mu_{n+1}=\left\langle A v_{n}, v_{n}\right\rangle \cdot\left\|v_{n}\right\|^{-2}, \quad v_{n+1}+\mu_{n+1}^{-1} A v_{n},
$$

where the initial approximation $v_{0} \in X$ satisfies the conditions $v_{0}$ ker $A,\left\|v_{0}\right\|=1$; (iii) the Birger method:

$$
q_{n+1}=\left\langle A y_{n}, y_{n}\right\rangle \cdot\left\|A y_{n}\right\|^{-2}, \quad y_{n+1}=q_{n+1} A y_{n},
$$

where the starting approximation $y_{0} \in X$ is such that $y_{0} \notin \operatorname{ker} A$, $\left\|y_{0}\right\|=1$.

Lemma $1:$ Let $A: X \rightarrow X$ be a linear non-negative and self-adjoint operator, $u_{0}, v_{0}$, $y_{0} \in X$ the starting approximations of the methods $(1)-(3)$, respectively, such that $u_{0}=v_{0}$ $=y_{0}, u_{0} \notin \operatorname{ker} A,\left\|u_{0}\right\|=1$. Then:

$$
\begin{array}{ll}
\text { (i) } & \mu_{n}>0, q_{n}>0, v_{n} \neq 0, y_{n} \neq 0 ; \\
\text { (ii) } & \mu_{n} \leqq \alpha_{n} \leqq q_{n}^{-1} \leqq \mu_{n+1} \leqq \alpha_{n+1} \leqq q_{n+1}^{-1} ; \\
\text { (iii) } & v_{n+1}=\left(\prod_{i=1}^{n+1}\left(\alpha_{i} \mu_{i}^{-1}\right)\right) u_{n+1} ; \\
\text { (iv) } & y_{n: 1}=\left(\prod_{i=1}^{n+1} \alpha_{i} q_{i}\right) u_{n+1}
\end{array}
$$

for each $n \geqq 1$.

Proof: Since $A$ is non-negative and self-adjoint, $\langle A u, v\rangle^{2} \leqq\langle A u, u\rangle\langle A v, v\rangle$ for each $u, v \in X$. According to our assumption $u_{0} \notin$ ker $A$ we obtain

$$
0<\alpha_{1}{ }^{4}=\left\|A u_{0}\right\|^{4}=\left\langle A u_{0}, A u_{0}\right\rangle^{2} \leqq\left\langle A u_{0}, u_{0}\right\rangle\left\langle A^{2} u_{0}, A u_{0}\right\rangle=\mu_{1}\left\langle A^{2} u_{0}, . A u_{0}\right\rangle .
$$

Hence $\mu_{1}>0$ and $v_{1}=\mu_{1}^{-1} A v_{0}=\mu_{1}^{-1} A u_{0}=\alpha_{1} \mu_{1}^{-1} \cdot u_{1} \neq 0$. Now assume that $\mu_{k}>0, v_{k} \neq 0$ for each $k(k=1,2, \ldots n)$. We are going to show that $\mu_{n: 1}>0$ and $v_{n+1} \neq 0$. From

$$
0<a_{n+1}^{4}=\left\langle A u_{n}, A \dot{u}_{n}\right\rangle^{2} \leqq \mu_{n+1}\left\langle A^{2} u_{n}, A u_{n}\right\rangle
$$


we get that $\mu_{i+1}>0$. Moreover, $u_{n}=v_{n}\left\|v_{n}\right\|^{-1}$ and

$$
\begin{aligned}
v_{n+1} & =\mu_{n+1}^{-1} A v_{n}=\mu_{n+1}^{-1}\left\|v_{n}\right\| \cdot A\left(\frac{v_{n}}{\left\|v_{n}\right\|}\right)=\mu_{n+1}^{-1}\left\|v_{n}\right\| A u_{n}=\alpha_{n+1} \mu_{n+1}^{-1}\left\|v_{n}\right\| \cdot u_{n+1} \\
& =\alpha_{n+1} \mu_{n+1}^{-1} \alpha_{n} \mu_{n}^{-1}\left\|v_{n-1}\right\| \cdot u_{n+1}=\cdots=\left(\prod_{k=1}^{n+1}\left(\alpha_{k}, \mu_{k}^{-1}\right)\right) u_{n+1} \neq 0
\end{aligned}
$$

'The proofs of the equality (iv) and the facts.that $q_{n}>0, y_{n} \neq 0$ are quite similar.

$$
\begin{aligned}
\mu_{n} & =\left\langle A v_{n-1}, v_{n-1}\right\rangle .\left\|v_{n-1}\right\|^{-2}=\left\langle A u_{n-1}, u_{n-1}\right\rangle \leqq\left\|A u_{n-1}\right\| \cdot\left\|u_{n-1}\right\|=\left\|A u_{n-1}\right\|=\alpha_{n}, \\
q_{n}^{-1} & =\left\langle A y_{n-1}: y_{n-1}\right\rangle^{-1}\left\|A y_{n-1}\right\|^{2}=\left\|A u_{n-1}\right\|\left\langle A u_{n-1}, u_{n-1}\right\rangle^{-1}=\alpha_{n}\left(\alpha_{n} \mu_{n}^{-1}\right) \geqq \alpha_{n} .
\end{aligned}
$$

Furthermore, according to the generalized Schwarz inequality .

$$
\begin{aligned}
& q_{n}^{-1}=\left\langle A u_{n-1}, u_{n-1}\right\rangle^{-1}\left\|A u_{n-1}\right\|^{2} \leqq\left\langle A^{2} u_{n-1}, A u_{n-1}\right\rangle \cdot\left\|A u_{n}\right\|^{-4} \cdot\left\|A u_{n-1}\right\|^{2} \\
& \therefore={ }_{i}\left\langle A^{2} u_{n-1}, A u_{n-1}\right\rangle\left\|A u_{n-1}\right\|^{-2}=\left\langle A u_{n}, u_{n}\right\rangle=u_{n+1} .
\end{aligned}
$$

This completes the proof Lemma 1

Ieimmą 1 enables a slight extension of the corresponding results of $[11,12]$ for non-negative operators.

Theorem 1: Let $A: X \rightarrow X$ be a linear non-negative self-adjoint operator, $u_{0}, v_{0}$, $y_{0} \in X$ the starting approximation of the methods (1)-(3), respectively, such that

$$
u_{0}=v_{0}=y_{0}, \quad u_{0} \notin \operatorname{ker} A, \quad\left\|u_{0}\right\|=1 .
$$

Then the following conclusions are valid:

(i) If $u_{0}$ is such that $\dot{E}_{2} u_{0} \neq u_{0}$ for each $\lambda<\lambda_{1}$, then the sequences $\left(a_{n}\right),\left(\mu_{n}\right),\left(q_{n}^{-1}\right)$. are monotone increasing and converge to $\lambda_{1}$.

(ii) If $\lambda_{1}$ (not necessarily an isolated point of $\sigma(-A)$ with finile multiplicity) is an eigenvalue of $A$ and $u_{0}$ is such that $u_{0} \notin \operatorname{ker}\left(A-\lambda_{1} I\right)^{\perp}$, then $\left(\alpha_{n}\right),\left(\mu_{n}\right),\left(q_{n}{ }^{-1}\right)>\lambda_{1}$ as $n \rightarrow \infty$.

iii) If $\lambda_{1}$ is an isolated point of $\sigma(A)$ and $u_{0} \notin \operatorname{ker}\left(A-\lambda_{1} I\right)^{\perp}$, then euch of the following sequences $\left(u_{n}\right),\left(v_{n}\right),\left(y_{n}\right)$ converge to one of the eigenvectors of $A$ corresponding $t o \lambda_{1}$.

Under the additional hypothesis that $A$ is compact we shall prove that the methods (1) to (3) are convergent even in the case when the starting approximations $u_{0}, v_{0}, y_{0}$ are only different from zero.

We shall use the following

Lemma 2: Under the ussumptions of Lemma 1 the sequences $\left(\left\|v_{n}\right\|\right)$, ( $\left.\|y\|_{n}\right)$ are monotone increasing and decreasing, respectively, and convergent.

Prof : It relies on Temma 1 and the arguments of the proof of Iemma 2 [9] and [8], where the assumptions of positivity of $A$ is superfluous

Theorem 2: Let $A: X \rightarrow X$ be a linear non-negative and self-adjoint operator such that $A^{n_{0}}$ is compact for some positive integer $n_{0}$. Assume that the stariing approximations $u_{0}, v_{0}, y_{0}$ are such thut $u_{0}=v_{0}=y_{0}$ and $\left\|u_{0}\right\|=1$.

Then there is valid:

(i) The sequences $\left(a_{n}\right),\left(\mu_{n}\right),\left(q_{n}^{-1}\right)$ are monotone increasing and converge to some positive eigenvalue $\lambda^{*}$ of $A$.

(ii) The sequences $\left(u_{n}\right),\left(v_{n}\right),\left(y_{n}\right)$ converge to one of the eigenvectors corresponding $t^{o \cdot} \lambda^{*}$ 
Proof: According to i.emma 1 we have that $\left(\alpha_{n}\right),\left(\mu_{n}\right),\left(q_{n}^{-1}\right) \ngtr \lambda^{*}$ as $n \rightarrow \infty$ and $0<\lambda^{*} \leqq \lambda_{1}=\|A\|$. From (2) it follows that

$$
\left\|v_{n+1}-v_{n}\right\|^{2}=\left\|\mu_{n+1}^{-1} A v_{n}-v_{n}^{\prime}\right\|^{2}=\left\|v_{n+1}\right\|^{2}-j \mid v_{n} \|^{2}
$$

for each $n$. Put $z_{n}=v_{n+1}$ for each $n \geqq 1$. By Iemma 2 the sequence $\left(z_{n}-v_{n}\right)$ converges to 0 as $n \rightarrow \infty$. Moreover, the sequence $\left(z_{n}\right)$ is bounded and our hypotheses imply that $A$ is compact [20: ch. 12]. Hence $\left(z_{n}\right)$ contains a convergent subsequence $\left(z_{n_{k}}\right)$. Denote $v^{*}=\lim _{k \rightarrow \infty} z_{n_{k}}$. In view of (4) we conclude that $v_{n_{k}} \rightarrow v^{*}$. Since $\left(\left\|v_{n}\right\|\right)$ is monotone increasing and $v_{0}^{*} \neq 0$ we get that $v^{*} \neq 0$. But continuity of $A$ implies thät $A v^{*}=\lambda^{*} v^{*}$ and $\mu_{n} \nearrow \lambda^{*}=\left\langle A u^{*}, u^{*}\right\rangle \cdot\left\|u^{*}\right\|^{-2}$. From (4) it follows that $\left(v_{n}\right)$ is a Cauchy sequence. Since it contains a converging subsequence $\left(v_{n_{k}}\right)$, the whole sequence $\left(v_{n}\right)$ converges to $v^{*}$.

By Lemma 1

$$
v_{n}=\left(\prod_{i=1}^{n}\left(\alpha_{i} \mu_{i}^{-1}\right)\right) u_{n}, \quad y_{n}=\left(\prod_{i=1}^{n}\left(\alpha_{i} \varphi_{i}\right)\right) u_{n}=\left(\prod_{i=1}^{n}\left(\mu_{i} \alpha_{i}^{-1}\right)\right) u_{n}
$$

Since $\lim _{n \rightarrow \infty}\left\|v_{n}\right\|=\sup _{n=1,2 \ldots}\left\|v_{n}\right\|=\prod_{i=1}^{\infty}\left(a_{i} \mu_{i}^{-1}\right)$ is finite and positive, there exist $\lim _{n \rightarrow \infty} u_{n}=u^{*}$, $\lim _{n \rightarrow \infty} y_{n} \rightarrow \infty=y^{*}$ and

$$
\prod_{i=1}^{\infty}\left(\alpha_{i}^{\prime} \mu_{i}^{-1}\right) u^{*}=v^{*}, \cdot \prod_{i=1}^{\infty}\left(\alpha_{i} q_{i}\right) u^{*}=y^{*} .
$$

Hence $u^{*} \in \operatorname{ker}\left(A-\lambda^{*} I\right), y^{*} \in \operatorname{ker}\left(A-\lambda^{*} I\right)$ and $u^{*} \neq 0, y^{*} \neq 0$. Theorem 2 is proved

Using arguments similar to that of [8], one can extend the result of Theorem 2 to symmetrizahle compact operators.

Tet $B: X \rightarrow X, C: X \rightarrow X$ be linear self-adjoint operators, $\lambda_{0}$ an eigenvalue of $B$ such that $\lambda_{0} \notin \sigma(C), \lambda^{*}$ an eigenvalue of $C$. We shall say that $\lambda^{*}$ is nearest to $\lambda_{0}$ from the both sides if there exists a positive number $\varepsilon$ such that $\lambda^{*} \in J=\left(\lambda_{0}-\varepsilon, \lambda_{0}+\varepsilon\right)$ and $\lambda \in \sigma_{p}(C), \lambda \neq \lambda^{*}$ imply that $\lambda \notin J$, where $\sigma_{p}(C)$ denotes the point spectrum of $C$.

The following result is an extension of Theorem 4 of [12].

Theorem 3: Let $B: X \rightarrow X, C: X \rightarrow X$ be linear self-adjoint operators. Suppose that $i_{0}$ is an eigenvalue of $B, e_{0} \in \operatorname{ker}\left(B-\lambda_{0} I\right),\left\|e_{0}\right\|=1$ and that $\lambda_{0} \leftarrow \sigma(C)$. Let $\lambda^{*}$ be an eigenvalue of $C$ such that $\lambda^{*}$ is nearest to $i_{0}$ from the both sides. If $e_{0} \notin \operatorname{ker}\left(C-\lambda{ }^{*} I\right)^{1}$, then

$$
\left|\lambda^{*}-\lambda_{0}\right| \leqq\left\|\left(C-\lambda_{0} I\right) u_{n}\right\| \leqq \cdots \leqq\left\|\left(C-\lambda_{0} I\right) u_{0}\right\| \leqq\|B-C\|,
$$

where $\left(u_{n}\right)$ is defined by the Kellogg method (1) with $A=\alpha I-\left(C-\lambda_{0} I\right)^{2}, u_{0}=e_{0}$ and $\alpha$ is un arbitrary constant such that $\alpha>\left\|\left(C-\lambda_{0} I\right)^{2}\right\|$.

Proof: The operators $B, C$ are both bounded by the closed-graph theorem. Put $A=\alpha I-\left(C-\lambda_{0} I\right)^{2}$, where $\alpha>\left\|\left(C-\lambda_{0} I\right)^{2}\right\|_{1}$. Then $A$ is self-adjoint bounded and positive definite with the greatest isolated point $\lambda_{1}$ of $\sigma(A)$, where $\lambda_{1}=\alpha-\left(\lambda^{*}-\lambda_{0}\right)^{2}$. Put $C_{1}=C-\lambda_{0} I, \lambda=\lambda^{*}-\lambda_{0}, C_{2}=C-\lambda^{*} I$. We show that ker $\left(C_{1}-\lambda^{2} I\right)$ $\supset \operatorname{ker} C_{2}$. Suppose that $u \in \operatorname{ker} C_{2}$; this condition is equivalent to $C_{1} u=\lambda \cdot u$. Then $C_{1}^{2} u=C_{1}(\lambda u)=\lambda^{2} u$. Hence $u \in \operatorname{ker}\left(C_{1}^{2}-\lambda_{1}^{2} I\right)$ and we get that ker $\left(A-\lambda_{1} I\right)$ $\supset \operatorname{ker}\left(C-\lambda^{*} I\right)$. Therefore $\operatorname{ker}\left(A-\lambda_{1} I\right)^{\perp} \subset \operatorname{ker}\left(C-\lambda^{*} I\right)^{1}$ and hence $u_{0} \notin \operatorname{ker}(A$ $\left.-\lambda_{1} I\right)^{\perp}$. According to Theorem 3 of [11] $\mu_{n+1}=\left\langle A u_{n}, u_{n}\right\rangle \lambda_{\lambda_{1}}=\alpha-\left(\lambda^{*}-\lambda_{0}\right)^{2}$ : 
Hence $\left\langle\left(C-\lambda_{0} I\right) u_{n}, u_{n}\right\rangle \searrow\left(\lambda^{*}-\lambda_{0}\right)^{2}$ as $n \rightarrow \infty$. This conclusion implies that

$$
\begin{aligned}
\left|\lambda^{*}-\lambda_{0}\right| & \leqq\left\langle\left(C-\lambda_{0} I\right)^{2} u_{n}, u_{n}\right\rangle^{1 / 2}=\left\|\left(C-\lambda_{0} I\right) u_{n}\right\| \leqq\left\langle\left(C-\lambda_{0} I\right)^{2} u_{n-1}, u_{n-1}\right\rangle^{1 / 2} \\
& =\left\|\left(C-\lambda_{0} I\right) u_{n-1}\right\| \leqq \cdots \leqq\left\|\left(C-\lambda_{0} I\right) e_{0}\right\|=\left\|(C-B) e_{0}\right\| \leqq\|C-B\|,
\end{aligned}
$$

because $e_{0} \in \operatorname{ker}\left(B-\lambda_{0} I\right),\left\|e_{0}\right\|=1$ and $u_{0}=e_{0}$, which concludes the proof

We shall consider the condition concerning the starting approximations of the methods (1)-(3) which occurs in Theorem 1.

Theorem 4: Let $A: X \rightarrow X$ be a lineur non-negative self-adjoint operator, $u_{0} \in X$. Then the following assertions are equivalent:

$$
E_{\lambda} u_{0} \neq u_{0} \text { for euch } \lambda<\lambda_{1}
$$

$$
\left\|A^{n} u_{0}\right\|^{1 / n} \rightarrow \lambda_{1} \quad \text { as } n \rightarrow \infty \text {; }
$$

$$
\left\langle A^{n} u_{0}, u_{0}\right\rangle^{1 / n} \rightarrow \lambda_{1} \text { us } n \rightarrow \infty \text {. }
$$

Proof: We prove that (i) $\Rightarrow$ (ii) $\Rightarrow$ (iii) $\Rightarrow$ (i).

(i) $\Rightarrow$ (ii): Assume that $u_{0} \in X$ is such that $E_{i} u_{0} \neq u_{0}$ for each $\lambda<\lambda_{1}$. Let $\varepsilon$ be an arbitrary number such that $0<\varepsilon<\lambda_{1}$, where $\lambda_{1}$ denotes the lower upper bound of $\sigma(A)$. Since $\|A\|=\lambda_{1}$, the spectral theorem implies that

Hence

$$
\begin{gathered}
\lambda_{1}^{2 n}\left\|u_{0}\right\|^{2} \geqq\left\|A^{n}\right\|^{2} \cdot\left\|u_{0}\right\|^{2} \geqq\left\|A^{n} u_{0}\right\|^{2} \geqq\left\langle A^{2 n} u_{0}, u_{0}\right\rangle=\int_{0}^{\lambda_{1}} \lambda^{2 n} d\left\|E_{\lambda} u_{0}\right\|^{2} \\
\geqq \int_{\lambda_{1}-\varepsilon}^{\lambda_{1}} \lambda^{2 n} d\left\|E_{2} u_{0}\right\|^{2} \geqq\left(\lambda_{1}-\varepsilon\right)^{2 n}\left\|u_{0}-E_{\lambda_{1}-\varepsilon} u_{0}\right\|^{2}
\end{gathered}
$$

$$
\lambda_{1}-\varepsilon \leqq \liminf _{n \rightarrow \infty}\left\|A^{n} u_{0}\right\|^{1 / n} \leqq \limsup _{n \rightarrow \infty}\left\|A^{n} u_{0}\right\|^{1 / n} \leqq \lambda_{1}
$$

Therefore $\lim \left\|A^{n} u_{0}\right\|^{1 / n}=\lambda_{1}$.

' (ii) $\Rightarrow\left(\right.$ iii) $:$ Assume that $\lim _{n \rightarrow \infty} \|\left. A^{n} u_{0} !\right|^{1 / n}=\lambda_{1}$. We have that $\left\langle\cdot A^{n} u_{0}, u_{0}\right\rangle^{1 / n} \leqq\left\|A^{n} u_{0}\right\|^{1 / n}$ $\times\left\|u_{0}\right\|^{1 / n}$. On the other hand, the Reid inequality [17] implies that

$$
\left\|_{i} A^{n} u_{0}\right\|^{2} \leqq\left\|A^{n}\right\|\left\langle A^{n} u_{0}, u_{0}\right\rangle \leqq\|A\|^{n}\left\langle A^{n} u_{0}, u_{0}\right\rangle \leqq \hat{\lambda}_{1}{ }^{n}\left\langle A^{n} u_{0}, u_{0}\right\rangle .
$$

Therefore $\left\langle A^{n} u_{0}, u_{0}\right\rangle^{1 / n} \geqq \lambda_{1}^{-1}\left\|A^{n} u_{0}\right\|^{2 / n}$. Now our conclusion follows at once from the above inequalities.

(iii) $\Rightarrow$ (i): Assume that $\lim \left\langle A^{n} u_{0}, u_{0}\right\rangle^{1 / n}=\lambda_{-1}$ for some $u_{0} \in X, u_{0} \neq 0$. We prove that $E_{\lambda} u_{0} \neq u_{0}$ for each $\lambda^{n \rightarrow \infty}<\lambda_{1}$. Suppose conversely, then there exists $\lambda_{0}$ such that $\lambda_{0}<\lambda_{1}$ and $E_{\lambda} u_{0}=u_{0}$. Then we have $E_{i} u_{0}=E_{\lambda} E_{\lambda_{0}} u_{0}=E_{\lambda_{0}} u_{0}=u_{0}$ for cach $\lambda \geqq \lambda_{0}$. Therefore

Hence

$$
0 \leqq\left\langle A^{n} u_{0}, u_{0}\right\rangle=\int_{0}^{\lambda_{1}} \lambda^{n} d\left\|E_{\lambda} u_{0}\right\|^{2} \leqq \lambda_{0} \int_{0}^{\lambda_{0}} d\left\|E_{\lambda} u_{0}\right\|^{2}=\lambda_{0}{ }^{n}\left\|E_{\lambda_{0}} u_{0}\right\|^{2}=\lambda_{0}{ }^{n}\left\|u_{0}\right\|^{2} .
$$

and

$$
0 \leqq\left\langle A^{n} u_{0}, u_{0}\right\rangle^{1 / n} \leqq \lambda_{0}\left\|u_{0}\right\|^{2 / n}
$$

$$
\lim _{n \rightarrow \infty}\left\langle A^{n} u_{0}, u_{0}\right\rangle^{1 / n}=\lambda_{1} \leqq \lambda_{0}<\lambda_{1}
$$

a contradiction. Hence $b_{i} u_{0} \neq u_{0}$ for each $\lambda<\lambda_{1}$. This finishes the proof 
- Remark 1: The assertion (i) $\Rightarrow$ (ii) of Theorem 4 is stated in [13: chapt. 2]. We gave the proof of this result here only for the sake of completeness. Let us note that the conclusion (ii) of Lemma 1 was observed firstly by DANEŠ [6]. But our proof relies on quite another and much more simpler arguments than in [6].

Proposition 1: Under the assumptions of Theorem 4 suppose that $\operatorname{ker}(A-\mu I) \neq$ (0) for some $\mu \in\left(0, \lambda_{1}\right)$ and that $u_{0} \neq 0$.

Then $u_{0} \notin \operatorname{ker}\left(A^{-}-\mu I\right)$ if and only if there exist constants $\lambda_{0}, \lambda^{*}, \lambda_{0} \geqq \mu, \lambda^{*}<\mu$ such that $E_{\lambda_{0}} u_{0} \neq u_{0}$ and $E_{j \cdot \bullet}\left(u_{0}\right) \neq 0$.

Proof: It is sufficient to prove the converse assertion: $u_{0} \in \operatorname{ker}(A-\mu I)$ if and only if $E_{\lambda} u_{0}=u_{0}$ for each $i \geqq \mu$ and $E_{\lambda} u_{0}=0$ for each $\lambda<\mu$.

Assume that $k_{i}^{\top} u_{0}=u_{0}$ for each $\lambda \geqq \mu$ and $E_{\lambda} u_{0}=0$ for all $\lambda<\mu$. Jet $\varepsilon$ be such that $0<\varepsilon<\operatorname{Min}\left(\mu, \lambda_{1}-\mu\right)$. Then

$$
\begin{aligned}
& \left\|(A-\mu I) u_{0}\right\|^{2}=\int_{0}^{\lambda_{1}}(\lambda-\mu)^{2} d\left\|E_{\lambda} u_{0}\right\|^{2}=\lim _{\varepsilon \downarrow 0} \int_{0}^{\mu-\varepsilon}(\lambda-\mu)^{2} d\left\|E_{i} u_{0}\right\|^{2} \\
& +\lim _{\varepsilon \downarrow 0} \int_{\mu-\varepsilon}^{\mu+\varepsilon}(\lambda-\mu)^{2} d\left\|E_{\lambda} u_{0}\right\|^{2}+\lim _{\varepsilon \searrow 0, \mu+\varepsilon} \int_{\mu}^{\lambda_{1}}(\lambda-\mu)^{2} d \| E_{\lambda} u_{0} !^{2}=0 .
\end{aligned}
$$

Hencé $u_{0} \in \operatorname{ker}(A-\mu I)$. Assume now that $u_{0} \dot{\epsilon} \operatorname{ker}(A-\mu I)$. Then

$$
\begin{aligned}
0= & \left\|(A-\mu I) u_{0}\right\|^{2}=\int_{0}^{\mu-\varepsilon}(\lambda-\mu)^{2} d\left\|E_{i} u_{0}\right\|^{2} \\
& +\int_{\mu-\varepsilon}^{\mu+\varepsilon}(\lambda-\mu)^{2} d\left\|E_{\lambda} u_{0}\right\|^{2}+\int_{\mu+\varepsilon}^{\lambda_{2}}(\lambda-\mu)^{2} d\left\|E_{i} u_{0}\right\|^{2} .
\end{aligned}
$$

From this equality it follows that $E_{i} u_{0}$ is constant on the intervals $(0, \mu-\varepsilon],[\mu+\varepsilon$, $\lambda_{1}$ ) for each $\varepsilon>0$. The properties of the spectral family $\left\{E_{\lambda}\right\}$ imply that $E_{i} u_{0}=\lim _{x \downarrow 0} E_{i}$ $\times u_{0}=\dot{0}$ for each $\lambda \in[0, \mu)$ and $E_{\lambda} u_{0}=\lim _{\lambda>\lambda_{1}} E_{\lambda} u_{0}=u_{0}$ for each $\lambda \in\left[\mu, \lambda_{1}\right]$ as desired

\section{REFERENCES}

[1] Anprushriw, R. I.: On the approximate solution of $K$-positive eigenvalue problems ' $T^{\prime}(u)-\lambda S(u)=0$. J. Math. Anal. Appl. (1975), $511-529$.

[2] BIHGER. I. A.: Some mathematical methods of sölving of engineering problems (in Russian). Oborongiz: Moscow 1956.

[3] Bïckser, H.: On iterative method for solving nonlinear integral equations. Symp. on the numerical treatment of ordinary differential equations, integral and integrodifferential equations, Rome 1960. Birkhäuser Verlag: Basel-Stuttgart 1960.

[4] Colsatz. L.: Eigenwertaufgaben mit technischen Anwendungen. Akad. Verlagsgesẹllschaft: Ieipzig 1963.

[5] Conway, W. E., and J. W. Thoms: Free streamline problems and the Milne-Thomson integral equations. J. of Math. Phys. Sciences 8.(1974), 67-93.

[6] DANEŠ, J.: Local spectral theory and approximation of eigenvalues and eigenvectors of self-adjoint operators. $9^{\text {th }}$ Vinter School on Abstract Analysis, January 26-31, 1981, Srní, Crechoslovakia.

[7] КоLomÝ, J.: On convergences of the iterative methods. Comment. Math. Univ. Carolinae 1 (1960), $18-24$.

[8] KoroM , J.: On the solution of homogeneous functional equations in Hilbert space. Comment. Math. Univ. Carolinae 3 (1962), 36-47. 
[9] Korouŕ, J.: Approximate determination of eigenvalues and eigenvectors of self-adjoint operators. Ann. Pol. Math. 3S (1980), 153-158.

[10] Kolom Y, J.: Determination of eigenvalues and eigenvectors of self-adjoint operators. Mathematica 20 (1980), $53-58$.

[11] KoLowÝ, J.: On determination of eigenvalues and eigenvectors of self-adjoint operators. Appl. Mat. .6 (1981), $161-170$.

[12] Kolom: J.: An approximate method for determination of eigenvalues and eigenvectors of self-adjoint operators. C̆as. pěst. mat. $106(1981), 243-255$.

[13] KrassoselskiJ, M. A., and co-authors: Approximate solving of operator equations (in Russian). Nauka: Moscow 1969.

[14] $\mathrm{IARFK}_{\mathrm{AB}}$ I. : Iterations of linear bounded operators in non-self-aldjoint eigenvalue problems and Kellogg's iteration process. Czech. Math. J. 12 (1962), 536-554.

[15] Marchuк, G. I.: Numerical methods for computation of nuclear reactors (in Russian). Atomizdat: Moscow 1958 .

[16] Petrysiry, W. V.: On the eigenvalue problem $T(u)-j . S(u)=0$ with unbounded and symmetric operators T', S. Phil. I'rans. Royal Soc. London, Ser. A, Math. Phys. Sci., No 1130, 262 (1968), 413-458.

[17] ReID, W: T.: Symmetriezable completely continuous linear transformations in Hilbert space. Duke Nath. J. 19 (1951), $41-56$.

[18] Thomas, J. W.: A numerical solution of the exact irrational gravity-wave problem. Pre. print.

[19] Yosi1), K.: Functional Analysis. Springer-Verlag: Berlin-Göttingen-Heidelberg 1965.

[20] Zanfex, A. C.: Iinear analysis. North-Holland Publ. Co.: Amsterdam 1960.

Manuskripteingang: 15.04 .1982

\section{VERFASSER :}

Prof. Dr. Joser Kolom

Matematicky Ustav University Karlovy

18600 Praha 8 - Karlin, Sokolovska 83 